\title{
The absolute summability $(A)$ of ultraspherical series
}

\author{
Memoria di D. P. Gupta (ad Allahabad, India)
}

Summary. - This paper is devoted to the discussion of $|A|$ summability of ultraspherical series on a sphere. The first theorem also gives a new result for LAPLACE series in case $\lambda=\frac{1}{\mathbf{2}}$.

1. Summability $|A|$ of Fourier series was discussed by WhITTAKer [8] and Prasad [6, 7]. Starting with Prasad's results, Bosanquet [2] gave general conditions for the absolute ABEL summability of FourIer series. BHatr [1] has studied conditions under which LAPLACE series is $|A|$ sum. mable at a point on the surface of a sphere of unit radius. In the present paper I propose to discuss absolute summability $(A)$ of the ultraspherical series at a point of the sphere. The ordinary ABEL summability along with Fatou's extensions for the ultraspherical series has been recently dealt with by Brousse [3].

2. If $F(\theta, \varphi)$ be a function defined for the range $0 \leq \theta \leq \pi, 0 \leq \varphi \leq 2 \pi$ on a sphere $S$, then the ultraspherical series associated with this function is,

$$
F(\theta, \varphi) \circlearrowleft \frac{1}{2 \pi} \sum_{n=0}^{\infty}(n+\lambda) \iint_{S^{\prime}} \frac{P_{n}^{(\lambda)}(\cos \Upsilon) F\left(\theta^{\prime}, \varphi^{\prime}\right) d \sigma^{\prime}}{\left[\sin ^{2} \theta^{\prime} \sin ^{2}\left(\varphi-\varphi^{\prime}\right)\right]^{\frac{1}{2}-\lambda}}, \quad \lambda>0
$$

where

$$
\cos r=\cos \theta \cos \theta^{\prime}+\sin \theta \sin \theta^{\prime} \cos \left(\varphi-\varphi^{\prime}\right)
$$

and

$$
d \sigma^{\prime}=\sin \theta^{\prime} d \theta^{\prime} d \varphi^{\prime}
$$

Assuming the LeBESGUE integrability of the function

$$
F\left(\theta^{\prime}, \varphi^{\prime}\right)\left[\sin ^{2} \theta^{\prime} \sin ^{2}\left(\varphi-\varphi^{\prime}\right)\right]^{\lambda-\frac{1}{2}}
$$

on the surface of the sphere, KogbetLiantz [4] (See also ObRechroff [5]) has defined the spherical mean value of $F(\theta, \varphi)$ as follows:

$$
f(\boldsymbol{\Upsilon})=\frac{1}{3 \pi(\sin \Upsilon)^{2 \lambda}} \int_{C_{\gamma}} \frac{F\left(\theta^{\prime}, \varphi^{\prime}\right) d s^{\prime}}{\left[\sin ^{2} \theta^{\prime} \sin ^{2}\left(\varphi-\varphi^{\prime}\right)\right]^{\frac{1}{2}-2}}
$$


where the integral is taken along the small circle whose centre is $(\theta, \varphi)$ and whose curvilinear radius is $r$.

We can now write (2.1) as

$$
F(\theta, \varphi) \propto \sum_{n=0}^{\infty}(n+\lambda) \int_{0}^{\pi} f(\Upsilon) \sin ^{2 \lambda} \Upsilon P_{n}^{(\lambda)}(\cos \Upsilon) d \Upsilon
$$

Now

$$
\begin{aligned}
& S(x)=\int_{0}^{\pi} f(\Upsilon) \stackrel{\infty}{\Sigma}_{0}^{\infty}(n+\lambda) P_{n}^{(\lambda)}(\cos \Upsilon) x^{n} \sin ^{2 \lambda} \Upsilon d \Upsilon \\
& =\int_{0}^{\pi} f(r) \frac{\lambda\left(1-x^{2}\right)}{\left(1-2 x \cos r+x^{2}\right)^{\lambda+1}} \sin ^{2 \lambda} r d r
\end{aligned}
$$

since [See KogBetLianiz [4], relation (7)]

$$
\sum_{m=0}^{\infty}(m+\lambda) P_{m}^{(\lambda)}(\cos \Upsilon) x^{m}=\frac{\lambda\left(1-x^{2}\right)}{\left(1-2 x \cos \Upsilon+x^{2}\right)^{\lambda+1}}
$$

Therefore

$$
Q(x) \equiv S(x)-F(P)=\int_{0}^{\pi}\left[f(\Lambda)-f(0) \frac{\Gamma(\lambda)}{\Gamma\left(\frac{1}{2}\right) \Gamma\left(\frac{1}{2}+\lambda\right)}\right] \frac{\lambda\left(1-x^{2}\right) \sin ^{2 \lambda} \Upsilon d \Gamma}{\left(1-2 x \cos \Upsilon+x^{2}\right)^{\lambda+1}}
$$

We write

$$
\begin{aligned}
& {\left[f(\Upsilon)-\frac{\Gamma(\lambda)}{\Gamma\left(\frac{1}{2}\right) \Gamma\left(\frac{1}{2}+\lambda\right)} f(0)\right] \sin ^{2 \lambda} \Upsilon=\varphi(\Upsilon) ;} \\
& \Phi_{\alpha}(\Upsilon)=\frac{1}{\Gamma(\alpha)} \int_{0}^{\Gamma}(\Gamma-u)^{\alpha-1} \varphi(u) d u, \quad \alpha>0 ; \\
& \Phi(\Upsilon)=\varphi(\Upsilon) ; \\
& \Phi_{\alpha}(\Upsilon)=\frac{d}{d \Upsilon} \Phi_{\alpha+1}(\Upsilon), \quad(-1<\alpha<0) ;
\end{aligned}
$$

and

$$
\varphi_{\alpha}(\Upsilon)=\Gamma(\alpha+1) \Upsilon^{-\alpha} \Phi_{\alpha}(\Upsilon)
$$


We shall first prove the following theorem:

Treorem 1. - The ultraspherical series (2.1) is absolutely summable (A), if the integral

$$
\int_{0}^{n} \frac{\left|\varphi_{\alpha}(t)\right|}{t^{1+2 \lambda}} d t
$$

exists for some $\alpha \geq 0, \eta>0$ and $0<\lambda<1$.

3. For the proof of the above theorem we shall require the following lemmas:

LeMma 1. - For $\beta>\alpha \geq 0$,

$$
\Gamma(\beta+1+2 \lambda) \int_{0}^{n} \frac{\left|\Phi_{\beta}(t)\right|}{t^{1+2 \lambda+\beta}} d t \leq \Gamma(\alpha+1+2 \lambda) \int_{0}^{n} \frac{\mid \Phi_{\alpha}(t)}{t^{1+2 \lambda+\alpha}} d t
$$

Proof. - The proof shall be exactly similar to that given by BosanqueT [2, Lemma 2]. We have

$$
\begin{aligned}
\Gamma(\beta+1+ & 2 \lambda) \int_{0}^{n} \frac{\left|\Phi_{\beta}(t)\right|}{t^{1+2 \lambda+\beta}} d t=\frac{\Gamma(\beta+1+2 \lambda)}{\Gamma(\beta-\alpha)} \int_{0}^{n} \frac{d t}{t^{1+2 \lambda+\beta}}\left|\int_{0}^{t}(t-u)^{\beta-\alpha-1} \Phi_{\alpha}(u) d u\right| \\
& \leq \frac{\Gamma(\beta+1+2 \lambda)}{\Gamma(\beta-\alpha)} \int_{0}^{n} \frac{d t}{t^{1+2 \lambda+\beta}} \int_{0}^{t}(t-u)^{\beta-\alpha-1}\left|\Phi_{\alpha}(u)\right| d u= \\
& =\frac{\Gamma(\beta+1+2 \lambda)}{\Gamma(\beta-\alpha)} \int_{0}^{n} \mid \Phi_{\alpha}(u) d u \int_{u}^{n}(t-u)^{\beta-\alpha-1} t^{-1-2 \lambda-\beta} d t \\
& \leq \frac{\Gamma(\beta+1+2 \lambda)}{\Gamma(\beta-\alpha)} \int_{0}^{n} \frac{\left|\Phi_{\alpha}(u)\right| d u}{u^{1+2 \lambda+\alpha}} \int_{1}^{\infty}(v-1)^{\beta-\alpha-1} v^{-\beta-1-2 \lambda} d v \\
& =\Gamma(\alpha+2 \lambda+1) \int_{0}^{n} \frac{\left|\Phi_{\alpha}(u)\right|}{u^{1+2 \lambda+\alpha}} d u .
\end{aligned}
$$

LEMMA 2. - If $0<t<\frac{\pi}{2}, 0<x<1$ and $r$ is a non-negative integer, 
then

$$
\left|\frac{\partial^{r+1} k(x, t)}{\partial x \partial t^{r}}\right|\left\{\begin{array}{l}
\leq A t^{-r-2 \lambda-2} \\
\leq A(1-x)^{-2} t^{-r-2 \lambda}
\end{array}\right.
$$

where $A$ is independent of $x$ and $t$, and

$$
k(x, t)=\frac{1-x^{2}}{\left(1-2 x \cos t+x^{2}\right)^{2+1}} .
$$

Proof. - It is easily verified by iuduotion that

$$
\frac{\partial^{r}}{\partial t^{r}} k(x, t)=\left(1-x^{2}\right) \sum_{\mu=0}^{r} T_{\mu}(t) \frac{x^{r-\mu}(\sin t)^{r-2 \mu}}{\left(1-2 x \cos t+x^{2}\right)^{1+2+r-\mu}},
$$

where $T_{p}(t)$ is a trigonometrical polynomial.

Since $T_{\mu}(t),(\mu=0,1,2, \ldots r)$ and $\frac{(1-x)(x-\cos t)}{1-2 x \cos t+x^{2}}$ are bounded, we have

$$
\frac{\partial^{r+1}}{\partial x \partial t^{r}} k(x, t)=\sum_{\mu=0}^{r} O\left\{\frac{(\sin t)^{r-2 \mu}}{\left(1-2 x \cos t+x^{2}\right)^{1+\lambda+r-\mu}}\right\} .
$$

Now

$$
\left(1-2 x \cos t+x^{2}\right)=(x-\cos t)^{2}+\sin ^{2} t \geq \sin ^{2} t
$$

Therefore

$$
\begin{aligned}
\frac{\partial^{r+1}}{\partial x \partial t^{r}} k(x, t)= & \stackrel{\Sigma_{\mu=0}^{r}}{ } O\left\{(\sin t)^{-2 \mu+r-2(1+\lambda+r-\mu)}\right\} \\
& =O\left(t^{-r-2 \lambda-2)} .\right.
\end{aligned}
$$

On the other hand

$$
\left(1-2 x \cos t+x^{2}\right)=(1-x)^{2}+4 x \sin ^{2} \frac{t}{2} \geq(1-x)^{2}
$$

Consequently, by (3.2) and (3.3) we have

$$
\begin{aligned}
\frac{\partial^{r+1}}{\partial x \partial t^{r}} k(x, t) & =\sum_{\mu=0}^{r} O\left\{(1-x)^{-2}(\sin t)^{r-2 i p-2(\lambda+r-\mu)}\right\} \\
& =O\left\{(1-x)^{-2} \sum_{\mu=0}^{r}(\sin t)^{-r-2 \lambda}\right\} \\
& =\left\{(1-x)^{-2} t^{-r-2 \lambda}\right\}
\end{aligned}
$$

which completes the proof. 
4. Proof of Theorem 1. - From (2.3) and (3.1) we obtain

$$
\begin{aligned}
Q(x) & =\int_{0}^{\pi} \varphi(t) k(x, t) d t \\
& =\left(\int_{0}^{n}+\int_{n}^{\pi}\right) \varphi(t) k(x, t) d t \\
& =Q_{1}(x)+Q_{2}(x), \quad \text { say. }
\end{aligned}
$$

Following the lines of argument of Prasad $[6,7]$ it is sufficient to prove that

$$
\int_{0}^{1}\left|Q_{1}^{\prime}(x)\right| d x<\infty .
$$

Without loss of generality we can suppose $0<\eta<\frac{\pi}{2}$; and by Lemma 1 , we can suppose that $\alpha=h$ is a positive integer $\left(^{\mathrm{a}}\right)$. Now, integrating by parts, we get

$$
\begin{gathered}
\int_{0}^{1}\left|Q_{1}^{\prime}(x)\right| d x \leq \int_{0}^{1} d x \sum_{\rho=0}^{h}\left|\Phi_{p}(\eta) \frac{\partial^{\rho}}{\partial x \partial \eta^{\rho-1}} k(x, \eta)\right|+ \\
\int_{0}^{1} d x \int_{0}^{n}\left|\Phi_{h}(u) \frac{\partial^{h+1}}{\partial x \partial u^{h}} k(x, u) d u\right| \\
=\int_{0}^{1} d x \sum_{p=0}^{h}\left|\Phi_{p}(\eta) \frac{\partial \rho}{\partial x \partial \eta^{p-1}} k(x, \eta)\right|+\frac{1}{\Gamma(h+1)} \int_{0}^{1} d x\left|\int_{0}^{n} u^{h} \varphi_{h}(u) \frac{\partial^{h+1}}{\partial x \partial u^{h}} k(x, u) d u\right| \\
\left.=C+\frac{1}{\Gamma(h+1)} \int_{0}^{\eta}\left|\varphi_{h}(u)\right| d u \int_{0}^{1} u^{h} \frac{\partial^{h+1}}{\partial x \partial u^{h}} k x, u\right) d x \mid,
\end{gathered}
$$

where $C$ is a constant.

Now

$$
I=\int_{0}^{1} u^{h} \frac{\partial^{h+1}}{\partial x \partial u^{h}} k(x, u) d x
$$

(1) If $\alpha$ be not an integer, we can take $h=[\alpha]+1$. 


$$
=\int_{0}^{1-u}+\int_{1-u}^{0}=I_{1}+I_{2}, \quad \text { say. }
$$

From Lemma 2,

$$
\begin{aligned}
I_{1} & =O\left[\int_{0}^{1-u}(1-x)^{-2} u^{-2 \lambda} d x\right] \\
& =O\left(u^{-2 \lambda-1}\right) .
\end{aligned}
$$

And

$$
I_{2}=O\left[\int_{1-u}^{1} u^{-2 \lambda-2} d x\right]=O\left(u^{-2 \lambda-1}\right)
$$

Therefore

$$
I=O\left(u^{-2 \lambda-1}\right)
$$

Thus we find that

$$
\int_{0}^{n}\left|Q_{1}^{\prime}(x)\right| d x \leq C+\frac{1}{\Gamma(h+1)} \int_{0}^{n} \frac{\left|\varphi_{h}(u)\right|}{u^{1+2 \lambda}} d u,
$$

which completes the proof.

5. We now write

$$
\psi(\gamma)=\left[f(\gamma)-\frac{\Gamma(\lambda)}{\Gamma\left(\frac{1}{2}\right) \Gamma\left(\frac{1}{2}+\lambda\right)} f(0)\right](\sin \gamma)^{2 \lambda-1}
$$

Using the corresponding RIGMANN-LiodvilLE fractional integrals, we shall prove the following theorem which includes both the theorems of BHATT [1] as particular cases for $\lambda=\frac{1}{2}$.

THEоREM 2. - If for some $\alpha \geq 0,0<\lambda \leq \frac{1}{2}$, $\psi_{\alpha}(\gamma)$ is of bounded variation in $(0, \eta)$ and $\psi_{a}(\gamma) \rightarrow 0$ as $\gamma \rightarrow 0$, then the ultraspherical series of $F(\theta, \varphi)$ is absolutely summable $(A)$ at the point $P$.

The general line of argument in the proof will be similar to that of Bosanquet [2] in the case of Fourier series. We need the following lemmas. 
Lemma 3. [4, Lemma 3] - If $\varphi_{x}(t)$ is of bounded variation in $(0, \eta)$ for a given $\alpha \geq 0$, then $\varphi_{\beta}(t)$ is of bounded variation in $(0, \eta)$, for every $\beta>\alpha$.

LEMMA 4. - If $0<t<\frac{\pi}{2}, 0<x<1$ and $r$ is a non-negative integer, then

$$
\left|\frac{\partial^{r+1}}{\partial x \partial t^{r}} \xi(x, t)\right|\left\{\begin{array}{l}
\leq A t^{-r-2 \lambda-1} \\
\leq A(1-x)^{-2} t^{-r-2 \lambda+1}
\end{array}\right.
$$

where $A$ is independent of $x$ and $t$ and

$$
\xi(x, t)=\frac{\left(1-x^{2}\right) \sin t}{\left(1-2 x \cos t+x^{2}\right)^{\lambda+1}} .
$$

The proof is exactly similar to that of Lemma 2.

LEMMA 5. - If $0<t<\frac{\pi}{4}$ and $r$ is a non-negative integer, then

$$
\left|\int_{0}^{t} u^{r} \frac{\partial^{r+1}}{\partial x \partial u^{r}} \xi(x, u) d u\right|\left\{\begin{array}{l}
\leq A t^{-2 \lambda}, \sigma \leq x<1 \\
\leq A(1-x)^{-2} t^{2-2 \lambda} \text { for } 0<x<1
\end{array}\right.
$$

$\sigma$ being a fixed positive constant less than unity.

Proof. - When $r=0$, the second inequality is evidently true since,

$$
\left.\int_{0}^{t} O\left\{(1-x)^{-2} u^{-2 \lambda+1}\right\} d u=O[1-x)^{-2} t^{2-2 \lambda}\right] .
$$

To prove the first inequality $\left({ }^{1}\right)$ in the case $r=0$, we write

$$
\chi(x)=\int_{0}^{t} \xi(x, u) d u
$$

Therefore

$$
\frac{\partial \chi(x)}{\partial x}=\int_{0}^{t} \frac{\partial \xi(x, u)}{\partial x} d u
$$

Putting

$$
\Delta=1-2 x \cos t+x^{2}
$$

(1) I must thank DR. S. N. BHATT for his valuable suggestions in this connection. 


$$
\begin{gathered}
\chi(x)=\int_{0}^{t} \frac{\left(1-x^{2}\right) \sin u}{\Delta^{\lambda+1}} d u \\
=-\left[\frac{\left(1-x^{2}\right)}{2 \lambda x \Delta^{\lambda}}\right]_{0}^{t}=\frac{1+x}{2 \lambda x(1-x)^{2 \lambda-1}}-\frac{1-x^{2}}{2 \lambda x \Delta^{\lambda}} .
\end{gathered}
$$

Hence

$$
\begin{aligned}
\frac{\partial \chi(x)}{\partial x} & =\frac{\partial}{\partial x}\left[\frac{1+x}{2 \lambda x(1-x)^{2 \lambda-1}}-\frac{1-x^{2}}{2 \lambda x \Delta^{\lambda}}\right] \\
& =O\left[x^{-2}(1-x)^{-2 \lambda+1} \Delta-\lambda\right]
\end{aligned}
$$

after simplification,

$$
=O\left[\sin t(\sin t)^{-2 \lambda}\right]=O\left(t^{-2 \lambda}\right) .
$$

If $r>0$, integration by parts gives

$$
\int_{0}^{t} u^{r} \frac{\partial^{r+1}}{\partial x \partial u^{r}} \xi(x, u) d u=t^{r} \frac{\partial^{r}}{\partial x \partial t^{r-1}} \xi(x, t)-r \int_{0}^{t} u^{r-1} \frac{\partial^{r}}{\partial x \partial u^{r-1}} \xi(x, u) d u,
$$

and the result of the lemma follows by induction.

6. Proof of Theorem 2. - From (5.1) and (5.2), we have

$$
\begin{gathered}
Q(x)=\int_{0}^{\pi} \psi(t) \xi(x, t) d t \\
=\int_{0}^{\delta}+\int_{\delta}^{\pi}=Q_{1}(x)+Q_{2}(x), \quad \text { say. }
\end{gathered}
$$

We need now only prove that

$$
\int_{0}^{1}\left|Q_{1}^{\prime}(x)\right| d x<\infty
$$

Without loss generality we can suppose $0<\delta<\frac{\pi}{4}$ and by Lemma 3 we may suppose $\alpha=h$ to be a positive integer. 
D. P. GUPTA: The absolute summability $(A)$ of ultraspherical series 187

Integration by parts gives as before

$$
\begin{aligned}
\int_{0}^{1}\left|Q_{1}^{\prime}(x)\right| d x & \leq \int_{0}^{1} d x \sum_{\rho=0}^{h}\left|\Psi_{\rho}(\delta) \frac{\partial \rho}{\partial x \partial \delta_{\rho}-1} \xi(x, \delta)\right|+ \\
& +\int_{0}^{1} d x\left|\int_{0}^{\delta} \Psi_{h}(u) \frac{\partial^{h+1}}{\partial x \partial u^{h}} \xi(x, u) d u\right| \\
& \leq \int_{0}^{1} d x \sum_{\rho=0}^{h}\left|\Psi_{\rho}(\delta) \frac{\partial^{\rho}}{\partial x \partial \delta^{\rho}-1} \xi(x, \delta)\right|+ \\
& +\frac{1}{\Gamma(h+1)} \int_{0}^{1} d x \psi_{h(\delta)} \int_{0}^{\delta} u^{h} \frac{\partial^{h+1}}{\partial x \partial u^{h}} \xi(x, u) d u \mid+ \\
& +\frac{1}{\Gamma(h+1)} \int_{0}^{1} d x \int_{0}^{\delta}\left|\psi_{h}^{\prime}(\theta)\right| d \theta\left|\int_{0}^{\theta} u^{h} \frac{\partial^{h+1}}{\partial x \partial u^{h}} \xi(x, u) d u\right| \\
& \leq C+\frac{1}{\Gamma(h+1)} \int_{0}^{\delta}\left|\psi_{h}^{\prime}(\theta)\right| d \theta \int_{0}^{1} d x\left|\int_{0}^{\theta} u^{h} \frac{\partial^{h+1}}{\partial x \partial u^{h}} \xi(x, u) d u\right|
\end{aligned}
$$

by virtue of the first inequalities of Lemmas 4 and 5 with $\theta=\delta, C$ being a constant.

Also, Lemma $\overline{5}$ gives for $0<\theta<\delta$,

$$
\begin{aligned}
& \int_{0}^{1} d x\left|\int_{0}^{A} u^{h} \frac{\partial^{h+1}}{\partial x \partial u^{h}} \xi(x, u) d u\right| \\
& =\int_{0}^{1-\theta}+\int_{1-\theta}^{1} \\
& =\int_{0}^{1-\theta} O\left[(1-x)^{-2} \theta^{2-2} \lambda\right] d x+\int_{1-\theta}^{1} \theta^{-2 \lambda} O(1) d x \\
& =O(1) \text {. }
\end{aligned}
$$


Since

$$
\int_{0}^{\delta}\left|\psi_{h}^{\prime}(\theta)\right| d \theta<\infty
$$

(6.1) now follows from (6.2) and (6.3).

It now remains to show that

$$
\lim _{x \rightarrow 1-0} Q(x)=0
$$

This can be easily established by following the line of argument usually employed for FourIer series.

I am indebted to Prof. B. N. Prasad for his continuous valuable help during the preparation of this paper.

\section{REFERENCES}

[1] S. N. Bнatт, The absolute summability (A) of Laplace series, "Bull. Calcutta Math. Soc. " Vol. 49 (1957), 129-132.

[2] L. S. Bosanquer, The absolute summability (A) of Fourier series, "Proc. Edin. Math. Soc. s, (2), Vol. 4 (193ł), 12.17.

[3] P. Brousse, Série ultrasphérique et integrale de Poisson, *J. Rational Mech. Anal.", Vol. 5 (1956), 967.986.

[4] E. Kogвetwrantz, Recherches sur la sommabilité des séries ultrasphériques des moyennes aritmétiques, "Journal de Mathématiques \&, (9), Vol. 3 (1924), 107.187.

[5] N. OвRеонкоғ, Sur la sommation de la série ultrasphériques par la methode des moyennes arithmétiques, * Rend. del Cire. Mat. di Palermo ", Vol. 59 (19:56), 266-287.

[6] B. N. Prasad, The absolute summability (A) of Fourier series, "Proc. Fdin. Math. Soc.", (2), Vol 2 (1931), 129-134.

[7] - -, On the summability of Fourier series and the bounded variation of power series, "Proc. London Math. Soe.", (2), Vol. 36 (1933), 407-424.

[8] J. M. Whiтtaker, The absolute summability of Fouries series, "Proc. Edin. Math. Soc. *, (2), Vol. 2 (1930), 1-5. 